University of Nebraska - Lincoln

DigitalCommons@University of Nebraska - Lincoln

Ilya Fabrikant Publications

Research Papers in Physics and Astronomy

2007

Dissociative Electron Attachment on Surfaces and in Bulk Media

Ilya I. Fabrikant

University of Nebraska-Lincoln, ifabrikant@unl.edu

Follow this and additional works at: https://digitalcommons.unl.edu/physicsfabrikant

Part of the Physics Commons

Fabrikant, llya I., "Dissociative Electron Attachment on Surfaces and in Bulk Media" (2007). Ilya Fabrikant Publications. 3.

https://digitalcommons.unl.edu/physicsfabrikant/3

This Article is brought to you for free and open access by the Research Papers in Physics and Astronomy at DigitalCommons@University of Nebraska - Lincoln. It has been accepted for inclusion in llya Fabrikant Publications by an authorized administrator of DigitalCommons@University of Nebraska - Lincoln. 


\title{
Dissociative electron attachment on surfaces and in bulk media
}

\author{
Ilya I. Fabrikant \\ Department of Physics and Astronomy, University of Nebraska, Lincoln, Nebraska 68588-0111, USA
}

(Received 23 April 2007; published 25 July 2007)

\begin{abstract}
A theory of dissociative electron attachment to molecules condensed at surfaces and embedded within bulk media is developed. The theory of low-energy electron diffraction is used to obtain the width for electronmolecule resonance scattering in the condensed phase from the width for the gas phase. It is then employed for the calculation of dissociative attachment in the framework of the nonlocal complex potential theory. Specific calculations using the effective mass approximation are carried out for electron attachment to $\mathrm{CH}_{3} \mathrm{Cl}$ and $\mathrm{CF}_{3} \mathrm{Cl}$ molecules physisorbed on the surface of a $\mathrm{Kr}$ film. The role of image states and image-potential resonances is analyzed. The results show an increase by several orders of magnitude in the cross section for physisorbed molecules as compared with gas-phase molecules. This is in general agreement with the measured cross sections. However, the position of the peak in the cross section for $\mathrm{CH}_{3} \mathrm{Cl}$ is significantly shifted towards higher energies as compared to experiment [K. Nagesha et al., J. Chem. Phys. 114, 4934 (2001)], and the magnitude of the calculated cross section for $\mathrm{CF}_{3} \mathrm{Cl}$ at the surface is significantly higher than the measured value. Possible reasons for disagreements are analyzed.
\end{abstract}

DOI: 10.1103/PhysRevA.76.012902

PACS number(s): 34.50.Dy, 34.80.Ht, 68.49.Jk

\section{INTRODUCTION}

Electron-molecule collision processes play a significant role in many condensed-matter systems where the effects of environment are important and the electron-molecule scattering process can no longer be considered as a binary interaction event [1]. Examples include processes relevant to radiation chemistry [2], the process of ozone depletion in polar stratospheric clouds $[3,4]$, and environmental remediation [5]. At energies of a few $\mathrm{eV}$ and below, vibrational excitation and dissociative electron attachment (DEA) are the most important of the processes through which electrons deposit energy and induce chemical transformations.

In spite of recent significant progress in experimental studies of electron attachment to molecules condensed on surfaces and embedded in bulk media [3,4,6-15], theory is still lagging behind. This is not surprising in view of the very complex collision conditions that occur in the condensedmatter and surface environments. Several theoretical studies [6,16-20] have substantially improved our understanding of resonance scattering by simple diatomics in the presence of the surface environment. However, it is apparent that for more complicated targets and for more complicated processes completely $a b$ initio calculations are not practically feasible yet. This is particularly relevant to DEA processes leading to the charging of dielectric films and fragmentation of adsorbates.

The first attempts $[8,21-23]$ to describe electron attachment in condensed-matter environment were based on model approaches employing a simple description of electron wave functions in a crystal. The resonance $R$-matrix model for the electron-molecule interaction $[24,25]$ was combined with the continuum-medium approximation for the medium. The band structure of the crystal was taken into account using the effective-mass approximation [22,23]. Several observed features in electron attachment to physisorbed $\mathrm{CH}_{3} \mathrm{Cl}$ and $\mathrm{CF}_{3} \mathrm{Cl}$ molecules, particularly the strong enhancement of the DEA cross section as compared to the gas phase results, were explained. However, disagreements were found also. In particular, the theory strongly underestimates DEA cross sections for $\mathrm{CH}_{3} \mathrm{Cl}$ embedded in a $\mathrm{Kr}$ film, but at the same time overestimates cross sections for $\mathrm{CF}_{3} \mathrm{Cl}$ [23].

In practice the rare gas molecular films are usually deposited on a metal substrate. For thick enough films with adsorbed molecules far from the metal surface, the metal effects are negligible, but as the thickness decreases electron interaction with the metal starts to affect the resonance width and to influence substantially the attachment cross sections. A simple empirical calculation [8] explained the major features in the dependence of the attachment cross sections on the film thickness. However, accurate $a b$ initio calculations of this effect present another challenge.

In the present paper we reformulate the model approach $[22,23]$ in terms of the nonlocal complex potential theory, since the latter is better adjusted to the inclusion of nonspherical geometry, particularly for electron collisions with surfaces. We assume that the dielectric film is infinitely thick, so that the metal effects can be neglected. We also assume that the attaching molecules are either placed on the dielectric surface or buried in the film at a finite distance from the surface. Specific calculations involve a series of approximations, the most important of which is the effective mass approximation for the electron wave function in the film. The results of the calculations describe qualitatively and semiquantitatively the condensed-matter effects in DEA to the $\mathrm{CH}_{3} \mathrm{Cl}$ and $\mathrm{CF}_{3} \mathrm{Cl}$ molecules. We discuss then how the theory can be improved in order to achieve a better agreement with experiment.

The rest of the paper is organized as follows. In Sec. II we discuss the wave function for the electron interacting with the surface. In Sec. III we develop the method of calculation of the resonance width for the molecule placed on a surface or embedded in a medium. This is a crucial step for the description of the DEA process since it occurs through the formation of a temporary negative ion (resonance) state. The resonance width as a function of electron energy and nuclear geometry serves as an input for the DEA cross section cal- 
culations. They are described in Sec. IV where we discuss first some features in DEA cross sections related to the geometry of the scattering and the presence of the imagecharge potential, and then present results for the $\mathrm{CH}_{3} \mathrm{Cl}$ and $\mathrm{CF}_{3} \mathrm{Cl}$ molecules. Conclusions are given in Sec. V.

\section{ELECTRON WAVE FUNCTION}

To account for the condensed-matter effects in DEA, we use the theory of low-energy electron diffraction (LEED) [26]. After the LEED functions are obtained, they can be used for calculation of DEA cross sections. To show how to do this, we will make a connection between the gas-phase and condensed-phase wave functions. We choose the system of units $e=\hbar=1$, but keep the notation $m$ for the electron mass in vacuum.

\section{A. Gas-phase wavefunction}

In the gas-phase theory the initial (continuum) state of the electron is characterized by the momentum $\mathbf{K}$ and the wave function [27]

$$
\psi_{\mathbf{K}}(\mathbf{r})=(2 / \pi)^{1 / 2} \sum_{l l^{\prime} m} i^{l^{\prime}} Y_{l m}^{*}(\hat{\mathbf{K}}) Y_{l^{\prime} m}(\hat{\mathbf{r}}) u_{l^{\prime} l}^{m}(r),
$$

normalized to the $\delta$ function of $\mathbf{K}$. Equation (1) is written in the body frame where the quantization axis is taken along the molecular axis. Here $u_{l l^{\prime}}^{m}(r)$ are radial wavefunctions satisfying the boundary condition of the incident plus outgoing wave

$$
\begin{aligned}
u_{l l^{\prime}}(r) \sim & \frac{i}{2 K r}\left\{\exp [-i(K r-l \pi / 2)] \delta_{l l^{\prime}}\right. \\
& \left.-\exp [i(K r-l \pi / 2)] S_{l l^{\prime}}\right\}, \quad r \rightarrow \infty,
\end{aligned}
$$

where $S$ is the scattering matrix. We assume that the molecule has cylindrical symmetry so that $m$ is a good quantum number. This is certainly true for linear molecules and is a good approximation for molecules with $C_{3 v}$ symmetry, such as $\mathrm{CH}_{3} \mathrm{Cl}$ and $\mathrm{CF}_{3} \mathrm{Cl}$, which will be the main object of the present study. The electron capture into the resonance state is described by the capture amplitude $V_{\mathbf{K}}^{(G)}$ [28],

$$
V_{\mathbf{K}}^{(G)}=\langle b|H| \psi\rangle,
$$

where $H$ is the total electronic Hamiltonian, and the state vector $|b\rangle$ represents the intermediate negative-ion state. We use the index $G$ to emphasize that we are dealing with the gas-phase amplitude at this point. Note that the capture amplitude depends also on the nuclear geometry. In the present paper this geometry will be represented by one internuclear separation $R$ corresponding to the reaction coordinate.

The capture amplitude can be expanded in partial waves as [28]

$$
V_{\mathbf{K}}^{(G)}=\sum_{l=|\mu|}^{\infty} V_{l K}^{(G)} Y_{l \mu}^{*}(\hat{\mathbf{K}}),
$$

where $\mu=\Lambda_{r}-\Lambda_{t}, \Lambda_{t}$ being the projection of the angular momentum of the target molecule, and $\Lambda_{r}$ that of the resonant state.
The total decay width can be written as

$$
\Gamma^{(G)}(K)=2 \pi \rho_{f}^{(G)} \int\left|V_{\mathbf{K}}^{(G)}\right|^{2} d \hat{\mathbf{K}},
$$

where the density of states with our normalization is

$$
\rho_{f}^{(G)}=m K
$$

\section{B. The condensed-phase wave function}

For a description of DEA in condensed phases we start with the LEED function $[29,30]$. Consider the $z=0$ plane as an interface between the vacuum $(z<0)$ and a crystal $(z$ $>0)$. We assume that attaching molecules are lying in a plane at a distance $r_{s}>0$ from the interface. In particular we can have $r_{s}=0$ for molecules lying on the surface. Consider first the incident electron beam neglecting the electronmolecule interaction. Outside the surface the electron interacts with the medium by the image potential

$$
V(z)=\frac{C}{4\left(z-z_{0}\right)}, \quad C=\frac{\kappa-1}{\kappa+1},
$$

where $\kappa$ is the dielectric constant of the medium, and $z_{0}$ a positive phenomenological parameter introduced to make the image potential finite at the interface. Suppose first that the incident wave propagates from the vacuum toward the surface. We will call this wave the "plus" solution. In the vacuum the electron wave function is

$$
\Psi^{(+)}=\psi_{\mathbf{K}}(\mathbf{r})+\sum_{i} R_{i}^{(+)} \psi_{i}(\mathbf{r}),
$$

where $\psi_{\mathbf{K}}(\mathbf{r})$ is the incident wave obtained by solving the Schrödinger equation with the potential (7) with the boundary condition

$$
\psi_{\mathbf{K}}(\mathbf{r}) \sim(2 \pi)^{-3 / 2} e^{i\left(\mathbf{K} \cdot \mathbf{r}+\delta_{c}\right)}, \quad z \rightarrow-\infty,
$$

where $\delta_{c}=C /\left(4 K^{\perp}\right) \ln (2|z|)$ is the Coulomb asymptotic phase for the one-dimensional Coulomb-potential problem. The quantity $K^{\perp}$ is the perpendicular component of the incident wave vector.

The solutions $\psi_{i}(\mathbf{r})$ are reflected waves and behave as

$$
\psi_{i} \sim(2 \pi)^{-3 / 2} \exp \left[i\left(-\mathbf{K}_{i}^{\perp}+\mathbf{K}^{\|}+\mathbf{g}_{i}^{\|}\right) \cdot \mathbf{r}-i \delta_{c i}\right]
$$

at $z \rightarrow-\infty$. Here $\mathbf{g}_{i}^{\|}$is the surface projection of the reciprocallattice vector $\mathbf{g}_{i}$. The parallel component $\mathbf{K}^{\|}$of the incident wave vector and the perpendicular component of the scattered wave vector $\mathbf{K}_{i}^{\perp}$ are related by the energy conservation

$$
\left|\mathbf{K}_{i}^{\perp}\right|=\left(2 m E-\left|\mathbf{K}^{\|}+\mathbf{g}_{i}^{\|}\right|^{2}\right)^{1 / 2} .
$$

Inside the crystal $(z>0)$ the plus solution can be written as a linear combination of Bloch waves [30]

$$
\Psi^{(+)}=\sum_{j} T_{j}^{(+)} \psi_{j}^{B}(\mathbf{r})
$$

where $T_{j}^{(+)}$are transmission coefficients, 


$$
\psi_{j}^{B}=(2 \pi)^{-3 / 2} \exp \left[i\left(\mathbf{k}_{j}^{\perp}+\mathbf{K}^{\|}+\mathbf{g}_{j}^{\|}\right) \cdot \mathbf{r}\right] u_{j}(\mathbf{r}),
$$

where $u_{j}(\mathbf{r})$ is a periodic function which can be expanded in plane waves $e^{i \mathbf{g}_{i} \cdot \mathbf{r}}$. In principle, both the expansion coefficients and the perpendicular components of the wave vector $\mathbf{k}_{j}^{\perp}$ can be determined by solving the secular equation derived from the Schrödinger equation for the crystal potential. However, at very low electron energies, such that the de Broglie wavelength is large compared to the lattice constant, we can adopt the following simplified approach. We assume that only one Bloch wave with the wave vector $\mathbf{k}$ (and $\mathbf{g}_{j}$ $=0$ ) contributes to expansion (12), and the solution of the secular equation yields a connection between $\mathbf{k}$ and $E$ given by the effective mass approximation formula

$$
E=\epsilon_{0}+\frac{k^{2}}{2 m^{*}},
$$

where $\epsilon_{0}$ is the bottom of the crystal conduction band, and $m^{*}$ is the effective mass. Note that in the approximation of the infinitely thick film and for a long electron wavelength we can assume that the effective mass is isotropic. For the $\mathrm{Kr}$ film, which we will be mostly interested in, $\epsilon_{0}=-0.25 \mathrm{eV}$ and $m^{*}=0.42 m$ [31].

To find the reflection and transmission coefficients entering Eqs. (8) and (12), we will also assume that only one term (with $\mathbf{g}_{i}=0$ ) contributes to the Bloch-wave expansion of $u_{j}(\mathbf{r}$ ) in plane waves. In other words, we assume that variation of the wave function inside the crystal on the scale of the atomic potential can be neglected. Again, this is a reasonable assumption for the long electron wavelength.

In summary, the simplified expressions for the wave function $\Psi^{(+)}$is

$$
\begin{gathered}
\Psi^{(+)}=\psi_{\mathbf{K}}(\mathbf{r})+R_{\mathbf{K}}^{(+)} \psi_{\mathbf{K}^{-}}(\mathbf{r}), \quad z<0, \\
\Psi^{(+)}=(2 \pi)^{-3 / 2} T_{\mathbf{K}}^{(+)} \exp \left[i \mathbf{k}^{+} \cdot \mathbf{r}\right], \quad z>0,
\end{gathered}
$$

where $\mathbf{K}^{-}=-\mathbf{K}^{\perp}+\mathbf{K}^{\|}, \mathbf{k}^{+}=\mathbf{K}^{\|}+\mathbf{k}^{\perp}$, and the absolute value of $\mathbf{k}^{+}$is given by Eq. (14).

Total reflection is possible if the incident angle $\theta$ satisfies the condition

$$
\sin ^{2} \theta>\frac{m^{*}}{m}\left(1-\frac{\epsilon_{0}}{E}\right) .
$$

In this case the transmitted wave is evanescent and $k^{\perp}$ $=i\left|k^{\perp}\right|$. The reflection and transmission coefficients in Eqs. (15) and (16) can be found from simple matching. Factorize first function $\Psi^{(+)}(\mathbf{r})$ in the Cartesian coordinates

$$
\Psi^{(+)}(\mathbf{r})=(2 \pi)^{-3 / 2} \exp \left[i \mathbf{K}^{\|} \cdot \mathbf{r}\right] \Phi^{(+)}(z),
$$

where

$$
\Phi^{(+)}(z)= \begin{cases}\chi_{K^{\perp}}(z)+R_{\mathbf{K}}^{(+)} \chi_{-K^{\perp}}(z), & \text { if } z<0, \\ T_{\mathbf{K}}^{(+)} e^{i k^{\perp} z}, & \text { if } z>0 .\end{cases}
$$

Here $\chi_{K^{\perp}}(z)$ is the solution of the one-dimensional Schrödinger equation with potential (7) behaving as $e^{i K^{\perp} z+i \delta_{c}}$ at $z \rightarrow-\infty$. It can be expressed in terms of the standard Coulomb functions $F_{L}(\eta, \rho)$ and $G_{L}(\eta, \rho)[32]$ as

$$
\chi_{K}(z)=G_{0}(\eta, \rho)-i F_{0}(\eta, \rho),
$$

where $\rho=K\left(z_{0}-z\right), \eta=-C /(4 K)$. Now we write the matching conditions as [33]

$$
\begin{gathered}
\Phi\left(z=0^{-}\right)=\Phi\left(z=0^{+}\right), \\
\frac{1}{m}\left(\frac{d \Phi}{d z}\right)_{z=0^{-}}=\frac{1}{m^{*}}\left(\frac{d \Phi}{d z}\right)_{z=0^{+}} .
\end{gathered}
$$

The second equation guarantees the conservation of current. As a result we obtain

$$
T^{(+)}=\frac{2 i K^{\perp}}{i m v^{\perp} \chi_{-K^{\perp}}-\chi_{-K^{\perp}}^{\prime}}, \quad R^{(+)}=-\frac{i v^{\perp} m \chi_{K^{\perp}}-\chi_{K^{\perp}}^{\prime}}{i v^{\perp} m \chi_{-K^{\perp}}-\chi_{-K^{\perp}}^{\prime}},
$$

where $v^{\perp}=k^{\perp} / \mathrm{m}^{*}$ and the prime means the derivative in $z$. For the evanescent wave $v^{\perp}$ is pure imaginary. All functions in Eqs. (23) are taken at $z=0$.

In order to calculate the resonance width, we need a complete set of the electron wave functions. The second class of solutions corresponds to incident waves propagating from the medium toward the interface (we will call them "minus" solutions):

$$
\begin{gathered}
\Psi^{(-)}=(2 \pi)^{-3 / 2}\left[\exp \left(i \mathbf{k}^{-} \cdot \mathbf{r}\right)+R_{\mathbf{k}}^{(-)} \exp \left(i \mathbf{k}^{+} \cdot \mathbf{r}\right)\right], \quad z>0, \\
\Psi^{(-)}=T_{\mathbf{k}}^{(-)} \psi_{\mathbf{K}^{-}}(\mathbf{r}), \quad z<0,
\end{gathered}
$$

where $\mathbf{k}^{-}=-\mathbf{k}^{\perp}+\mathbf{K}^{\|}$. In this case the transmitted wave is evanescent if the angle of incidence $\theta_{k}$, associated with vector $\mathbf{k}^{-}$, satisfies the condition

$$
\sin ^{2} \theta_{k}>\left[\frac{m^{*}}{m}\left(1-\frac{\epsilon_{0}}{E}\right)\right]^{-1} .
$$

The reflection and transmission coefficients are given now by

$$
T^{(-)}=\frac{2 i v^{\perp}}{i v^{\perp} \chi_{-K^{\perp}}+\chi_{-K^{\perp}}^{\prime} / m}, \quad R^{(-)}=\frac{i v^{\perp} m \chi_{-K^{\perp}}-\chi_{-K^{\perp}}^{\prime}}{i v^{\perp} m \chi_{-K^{\perp}}+\chi_{-K^{\perp}}^{\prime}} .
$$

An important feature of the minus solution is that it remains physical even below the vacuum level as soon as the energy is above the bottom of the conduction band, $E>\epsilon_{0}$. Although the initial conditions typically correspond to the incident wave propagating from vacuum with $E>0$, intermediate negative-ion state can have a negative electron energy because of virtual excitations of the target. Therefore negative energies above $\epsilon_{0}$ also contribute to the decay width.

The third class of solutions corresponds to the image states [34] which are bound in the $z$ direction but freely propagate on the surface. They exist due to the long-range image-charge potential, Eq. (7), and are possible even when the bottom of the conduction band lies below the vacuum level (such as, for example, in $\mathrm{Kr}$ and $\mathrm{Xe}$ ). This happens because the component of the electron energy parallel to the 


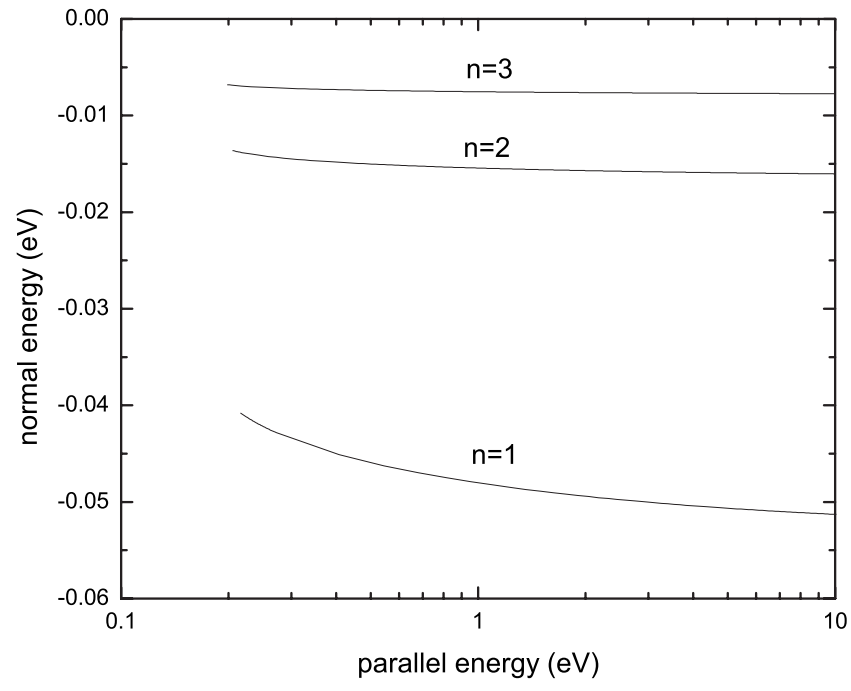

FIG. 1. Dependence of the energy of the image states in $\mathrm{Kr}$ on $\left(K^{\|}\right)^{2}$.

surface, $E^{\|}=\left(K^{\|}\right)^{2} / 2 m^{*}$, can be greater than the total energy $E$ relative to the bottom of the conduction band, so that $E$ $-E^{\|}<\epsilon_{0}$.

To analyze properties of these states let us write down the corresponding wave function $\Psi^{(s)}(\mathbf{r})$ in the form

$$
\Psi^{(s)}(\mathbf{r})=(2 \pi)^{-1} \exp \left[i \mathbf{K}^{\|} \cdot \mathbf{r}\right] \Phi^{(s)}(z),
$$

where

$$
\Phi^{(s)}(z)= \begin{cases}\chi_{\alpha}(z), & \text { if } z<0, \\ e^{-\kappa z}, & \text { if } z>0,\end{cases}
$$

where $\alpha^{2}=-\left(K^{\perp}\right)^{2}, \kappa^{2}=-\left(k^{\perp}\right)^{2}$, so that the relation between $\alpha$ and $\kappa$ is

$$
\frac{1}{m}\left[-\alpha^{2}+\left(K^{\|}\right)^{2}\right]=2 \epsilon_{0}+\frac{1}{m^{*}}\left[-\kappa^{2}+\left(K^{\|}\right)^{2}\right] .
$$

For each $K^{\|}$the spectrum of $\alpha$ is determined from the matching equation

$$
\frac{1}{m} \frac{\chi_{\alpha}^{\prime}}{\chi_{\alpha}}=-\frac{1}{m^{*}} \kappa
$$

Although the left-hand side of this equation has an infinite number of zeroes and poles, the solution is possible only for high enough $K^{\|}$because of the condition $\kappa^{2}>0$, or

$$
\left(K^{\|}\right)^{2}\left(m-m^{*}\right)+m^{*}\left(\alpha^{2}+2 m \epsilon_{0}\right)>0 .
$$

In Fig. 1 we plot $\alpha^{2}$ as a function of $\left(K^{\|}\right)^{2}$ for solid $\mathrm{Kr}$, whereby $\epsilon_{0}=-0.25 \mathrm{eV}, \kappa=1.91, m^{*}=0.42 m$, and parameter $z_{0}=2.955$ a.u. is chosen to reproduce the correct surface polarization energy $-0.72 \mathrm{eV}$ [35] for a $\mathrm{Kr}$ film. The infinite Coulomb spectrum appears at $\left(K^{\|}\right)^{2}=0.01307$ a.u., but for lower states higher values of $\left(K^{\|}\right)^{2}$ are necessary. For example, the $n=3$ state appears at $\left(K^{\|}\right)^{2}=0.0146$ a.u., the $n=2$ at $\left(K^{\|}\right)^{2}=0.0151$ a.u., and the ground state $n=1$ at $\left(K^{\|}\right)^{2}$ $=0.0159$ a.u.
We conclude that for a $\mathrm{Kr}$ surface the image states start to contribute to the decay width at rather low energies, above $0.18 \mathrm{eV}$, but they are very weakly bound. For the width calculation the solution $\chi_{\alpha_{n}}(z)$ should be normalized. This is achieved by calculation of the normalization constant $A_{n}$ from the condition

$$
A_{n}^{2}\left(\int_{-\infty}^{0}\left|\chi_{\alpha_{n}}(z)\right|^{2} d z+\frac{B_{n}^{2}}{2 \kappa}\right)=1,
$$

where $B_{n}=\chi_{\alpha_{n}}(0)$. Note that the image states exist for each total energy $E$ above the lower bound. The corresponding value of $K_{n}^{\|}$is determined from $2 m E=-\alpha_{n}^{2}+\left(K_{n}^{\|}\right)^{2}$.

\section{CONDENSED-PHASE WIDTH}

We will modify now the gas-phase capture amplitude, Eq. (4), to incorporate the surface effects. First we expand each plane wave in Eqs. (16) and (24) in spherical harmonics

$$
e^{i \mathbf{k} \cdot \mathbf{r}}=4 \pi \sum_{l m} i^{l} j_{l}(k r) Y_{l m}^{*}(\hat{\mathbf{k}}) Y_{l m}(\hat{\mathbf{r}}) .
$$

For evanescent waves with $\left(k^{\perp}\right)^{2}<0$ this expansion should be generalized. As was shown by Tong [26], in this case $Y_{l m}^{*}(\hat{\mathbf{k}})$ should be defined as (for $m \geqslant 0$ )

$$
Y_{l m}^{*}(\hat{\mathbf{k}})=(-1)^{m}\left[\frac{(2 l+1)}{4 \pi} \frac{(l-m) !}{(l+m) !}\right]^{1 / 2} P_{l}^{m}\left(\frac{k^{\perp}}{k}\right) e^{-i m \phi},
$$

where $P_{l}^{m}(z)$ is the analytical continuation of the associated Legendre functions into the complex plane. Note that $Y_{l m}^{*}(\hat{\mathbf{k}})$ is not the complex conjugated of $Y_{l m}(\hat{\mathbf{k}})$, if $\left(k^{\perp}\right)^{2}<0$. To emphasize this, we will be calling $Y_{l m}^{*}(\hat{\mathbf{k}})$ the adjoint of $Y_{l m}(\hat{\mathbf{k}})$.

According to Eq. (1) the electron-molecule interaction transforms each spherical wave $j_{l}(k r) Y_{l m}(\hat{\mathbf{r}})$ into

$$
\sum_{l^{\prime}} Y_{l^{\prime} m}(\hat{\mathbf{r}}) u_{l^{\prime} l}^{m}(r)
$$

Accordingly, the capture amplitude in the gas phase, Eqs. (3) and (4), is transformed into

$$
V_{\mathbf{K}}^{(+)}=T_{\mathbf{K}}^{(+)} e^{i k^{\perp}} r_{s} V_{\mathbf{k}^{+}}^{(G)}
$$

for the wave propagating from vacuum to the interface (plus solution),

$$
V_{\mathbf{k}}^{(-)}=e^{-i k^{\perp}} r_{s} V_{\mathbf{k}^{-}}^{(G)}+e^{i k^{\perp}} r_{s} V_{\mathbf{k}^{+}}^{(G)} R_{\mathbf{k}}^{(-)}
$$

for the minus solution, and

$$
V_{n}^{(s)}=(2 \pi)^{1 / 2} A_{n} B_{n} e^{-\kappa_{n} r_{s}} V_{\mathbf{k}}^{(G)}
$$

for the image-state solution. Again, for evanescent waves $V_{\mathbf{k}}^{(G)}$ should be understood as the analytical continuation of Eq. (4). Equations (37)-(39) assume that the part $\langle b| H$ of the matrix element (3) is not affected by the presence of the medium. This is a reasonable assumption since the shape of 
the virtual electron orbital corresponding to the resonance state is mainly determined by the electron-molecule interaction. The energy of the resonance state is significantly modified, however, due to the polarization of the medium by the negative ion. As in our previous $R$-matrix calculations $[22,23]$, this energy modification is included by a uniform shift of the anion potential energy curve. Equations (37)-(39) also imply that the molecule lies completely in the region $z$ $>0$. In practice it is important to know how the capture amplitude varies with the distance $r_{s}$ from the interface to the molecule, therefore it is reasonable to obtain the surface case by a gradual decrease of $r_{s}$ with reaching the limit $r_{s}=0$ and assuming that the electron wave function in the continuum does not vary significantly on the scale of the order of the size of the molecule. This assumption is valid for low-energy electrons.

The total decay width can be separated into three parts: the plus-solution contribution

$$
\Gamma^{(+)}=2 \pi \rho_{f} \int\left|V_{\mathbf{K}}^{(+)}\right|^{2} d \hat{\mathbf{K}}, \quad \rho_{f}=m K,
$$

where the integration is limited by the condition $K^{\perp}$ $=K \cos \theta_{K}>0$, the minus-solution contribution

$$
\Gamma^{(-)}=2 \pi \rho_{f} \int\left|V_{\mathbf{k}}^{(-)}\right|^{2} d \hat{\mathbf{k}}^{+}, \quad \rho_{f}=m^{*} k,
$$

where the integration is limited by the condition $k^{\perp}$ $=k \cos \theta_{\mathbf{k}^{+}}>0$, and the image-state-solution contribution

$$
\Gamma^{(s)}=\sum_{n}\left(2 \pi A_{n} B_{n}\right)^{2} e^{-2 \kappa_{n} r_{s}} \int\left|V_{\mathbf{k}}^{(s)}\right|^{2} \rho_{n} d \phi_{\mathbf{k}},
$$

where $\rho_{n} d \phi_{\mathbf{k}}$ is the density of the image states

$$
\begin{gathered}
\rho_{n} d \phi_{\mathbf{k}}=\int d \mathbf{K}^{\|} \delta\left[E-E_{n}\left(K^{\|}\right)\right] \\
=K_{n}^{\|}\left(\frac{d E}{d K^{\|}}\right)^{-1} K_{n}^{\|} d \phi_{\mathbf{k}},
\end{gathered}
$$

where the derivative is determined from the equation

$$
2 m E=-\alpha^{2}\left(K^{\|}\right)+\left(K^{\|}\right)^{2} .
$$

Therefore

$$
\rho_{n}=m\left(1-\frac{d \alpha_{n}^{2}}{d\left(K^{\|}\right)^{2}}\right)^{-1} .
$$

Although formally $\alpha^{2}$ as a function of $\left(K^{\|}\right)^{2}$ has a square-root singularity at the threshold, in general it varies very slowly (as can be seen from Fig. 1), so that $\rho_{n}$ is practically a constant, as typical for the density of surface states.

The validity of Eq. (41) for the minus-solution contribution is limited by the range of energies where the effectivemass relation between energy and momentum, Eq. (14), holds. In fact this region lies very close to the bottom of the conduction band. In solid $\mathrm{Kr}$, for example, deviations from Eq. (14) are seen already above $1.2 \mathrm{eV}$, whereas in DEA calculations substantially higher energies are involved, par- ticularly when the resonance shift is computed. The simplest way to correct Eq. (41) is to multiply it by the factor

$$
\Lambda=\rho_{f} / m^{*} k,
$$

where $\rho_{f}$ is the actual density of states. This modification does not take into account the correction to the matrix element due to the breakdown of the effective mass approximation. However, Michaud et al. [36] showed that the rate of inelastic processes in rare-gas films is mostly determined by the density of states in the conduction band. This allows direct observation $[36,37]$ of the density of states in experiments of phonon excitation in electron scattering from raregas films. In our calculations for DEA to molecules physisorbed on solid $\mathrm{Kr}$ we used the density of states calculated by Bacalis et al. [38] with the relativistic augmented-planewave method.

The total decay width is

$$
\Gamma=\Gamma^{(+)}+\Gamma^{(-)}+\Gamma^{(s)} .
$$

In order to express it in terms of the gas-phase amplitudes, we should recall that the amplitude $V_{\mathbf{K}}^{(G)}$ was defined in the body (molecular) frame, whereas all other quantities entering Eqs. (37)-(39) are defined in the laboratory frame with the $z$ axis normal to the interface. Therefore the gas-phase amplitudes should be transformed first into the laboratory frame by the use of the Wigner rotation matrix $D_{\mu m}^{l}(\hat{\mathbf{R}})$, where the unit vector $\hat{\mathbf{R}}$ with spherical angles $\beta$ and $\phi_{0}$ characterizes the orientation of the molecule. Since the molecule is treated as pseudodiatomic, as described in Sec. II A, the third Euler angle is 0 . The transformation from the body frame to the laboratory frame is

$$
Y_{l \mu}(\hat{\mathbf{k}}) \hat{\mathbf{R}}=\sum_{m} Y_{l m}(\hat{\mathbf{k}}) D_{\mu m}^{l}(\hat{\mathbf{R}})
$$

with a similar equation for the adjoint spherical harmonics. Since angles $\beta$ and $\phi_{0}$ are always real, we do not need to generalize the rotation matrix for the evanescent wave. Substituting Eqs. (4), (37)-(39), and (49) into Eqs. (40)-(42) we obtain

$$
\begin{aligned}
& \Gamma^{(+)}(\hat{\mathbf{R}})=2 \pi m K \sum_{l l^{\prime}} V_{l k}^{(G)} V_{l^{\prime} k}^{(G)} \sum_{m} D_{\mu m}^{l^{*}}(\hat{\mathbf{R}}) D_{\mu m}^{l^{\prime}}(\hat{\mathbf{R}})
\end{aligned}
$$

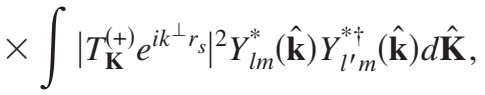

where "*†" means the complex conjugate of the adjoint,

$$
\begin{aligned}
& \Gamma^{(-)}(\hat{\mathbf{R}})=2 \pi m^{*} k \sum_{l l^{\prime}} V_{l k}^{(G)} V_{l^{\prime} k}^{(G) *} \sum_{m} D_{\mu m}^{l^{*}}(\hat{\mathbf{R}}) D_{\mu m}^{l^{\prime}}(\hat{\mathbf{R}}) \\
& \times \int\left[e^{-i k^{\perp} r_{s}}(-1)^{l+\mu}+e^{i k^{\perp} r_{s}} R_{\mathbf{k}}^{(-)}\right]\left[e^{i k^{\perp} r_{s}}(-1)^{l^{\prime}+\mu}\right.
\end{aligned}
$$

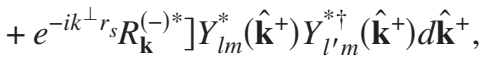




$$
\begin{aligned}
\Gamma^{(s)}(\hat{\mathbf{R}})= & (2 \pi)^{2} \sum_{n}\left(A_{n} B_{n}\right)^{2} e^{-2 \kappa_{n} r_{s}} \sum_{l l^{\prime}} V_{l k}^{(G)} V_{l^{\prime} k}^{(G)^{*}} \sum_{m} D_{\mu m}^{l^{*}}(\hat{\mathbf{R}}) \\
& \times D_{\mu m}^{l^{\prime}}(\hat{\mathbf{R}}) 2 \pi Y_{l m}^{*}(\hat{\mathbf{k}}) Y_{l^{\prime} m}^{* \dagger}(\hat{\mathbf{k}}) .
\end{aligned}
$$

In all equations the double sum in $m$ is reduced to the single sum because of the integration over $\phi_{\mathbf{K}}$.

We will consider now two simplifications of these general equations. First, let us assume that only one term with $l=l_{r}$ contributes to expansion (4). This is a typical approximation for low-energy resonance scattering by nonpolar molecules. For example, the $\Pi_{g}$ resonance in $e-\mathrm{N}_{2}$ scattering is dominated by the $d$ wave. Then from Eqs. (50) and (51) we obtain

$$
\begin{aligned}
& \Gamma^{(+)}(\hat{\mathbf{R}})=2 \pi m K\left|V_{l_{r} k}^{(G)}\right|^{2} \sum_{m}\left|D_{\mu m}^{l_{r}}(\hat{\mathbf{R}})\right|^{2} \\
& \times \int\left|T_{\mathbf{K}}^{(+)} e^{i k^{\perp} r_{s}} Y_{l_{r} m}^{*}(\hat{\mathbf{k}})\right|^{2} d \hat{\mathbf{K}}, \\
& \Gamma^{(-)}(\hat{\mathbf{R}})=2 \pi m^{*} k\left|V_{l_{r} k}^{(G)}\right|^{2} \sum_{m}\left|D_{\mu m}^{l_{\mu}^{*}}(\hat{\mathbf{R}})\right|^{2} \int \mid e^{-i k^{\perp} r_{s}(-1)^{l_{r}+\mu}} \\
& +\left.e^{i k^{\perp} r_{s} R_{\mathbf{k}}^{(-)}}\right|^{2}\left|Y_{l_{r} m}^{*}\left(\hat{\mathbf{k}}^{+}\right)\right|^{2} d \hat{\mathbf{k}}^{+}, \\
& \Gamma^{(s)}(\hat{\mathbf{R}})=(2 \pi)^{2} \sum_{n}\left(A_{n} B_{n}\right)^{2} \rho_{n} e^{-2 \kappa_{n} r_{s}}\left|V_{l_{r} k}^{(G)}\right|^{2} \\
& \times \sum_{m} 2 \pi\left|D_{\mu m}^{l_{r}}(\hat{\mathbf{R}}) Y_{l m}^{*}(\hat{\mathbf{k}})\right|^{2} .
\end{aligned}
$$

The other simplification corresponds to the randomly oriented molecules. In this case we have to average over all directions of $\hat{\mathbf{R}}$. Using the orthogonality of the Wigner's functions

$$
\frac{1}{4 \pi} \int D_{\mu m}^{l^{*}}(\hat{\mathbf{R}}) D_{\mu m^{\prime}}^{l^{\prime}}(\hat{\mathbf{R}}) \sin \beta d \beta d \phi_{0}=\frac{1}{2 l+1} \delta_{l l^{\prime}} \delta_{m m^{\prime}},
$$

we obtain

$$
\begin{aligned}
& \Gamma^{(+)}=\frac{m K}{2} \sum_{l}\left|V_{l k}^{(G)}\right|^{2} \int\left|T_{\mathbf{K}}^{(+)} e^{i k^{\perp} r_{s}}\right|^{2} \frac{4 \pi}{2 l+1} \sum_{m}\left|Y_{l m}^{*}(\hat{\mathbf{k}})\right|^{2} d \hat{\mathbf{K}},
\end{aligned}
$$

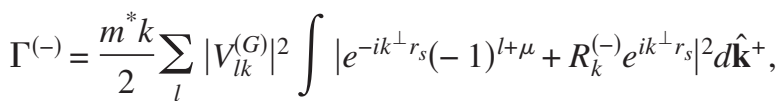

$$
\Gamma^{(s)}=(2 \pi)^{2} \sum_{n}\left(A_{n} B_{n}\right)^{2} \rho_{n} e^{-2 \kappa_{n} r_{s}} \sum_{l}\left|V_{l k}^{(G)}\right|^{2} \frac{2 \pi}{2 l+1} \sum_{m}\left|Y_{l m}^{*}(\hat{\mathbf{k}})\right|^{2} .
$$

Note that the sum over $m$ in Eqs. (57) and (59) cannot be simplified by using the addition theorem for spherical harmonics because the vector $\hat{\mathbf{k}}$ is generally complex.

\section{DISSOCIATIVE ATTACHMENT}

\section{A. General considerations}

Dissociative attachment cross section can be obtained by solving an inhomogeneous Schrödinger equation with a nonlocal complex potential $[39,40]$ whose kernel can be constructed from the width $\Gamma$ as a function of electron energy $E$ and internuclear separation $R$, and the shift function

$$
\Delta(E, R)=\frac{1}{2 \pi} P \int d E^{\prime} \frac{\Gamma\left(E^{\prime}, R\right)}{E-E^{\prime}},
$$

where $P$ stands for the principal value. For calculation of the shift we employ $\Gamma(E, R)$ with the correction factor (47) taking into account the actual density of states in the film. The gas-phase $\Gamma(E, R)$ is extrapolated above $E=5 \mathrm{eV}$ using an exponential function as usually suggested [39].

The inhomogeneous term in the Schrödinger equation with the nonlocal potential is proportional to the capture amplitude $V_{\mathbf{K}}(R)$, where we have introduced the explicit dependence of the capture amplitude on the internuclear separation $R$. For the electron beam incident from the vacuum we choose $V_{\mathbf{K}}^{(+)}(R)$ from Eq. (37). In the Franck-Condon approximation used within the framework of the quasiclassical theory [40] the DA cross section can be factorized as

$$
\sigma_{v \mathbf{K}}=\left|V_{\mathbf{K}}^{(+)}\left(R_{v}\right)\right|^{2} a_{v},
$$

where $v$ is the quantum number of the initial vibrational state, $R_{v}$ is the Franck-Condon point for the electron transition from the initial neutral state to the anion state, and the parameter $a_{v}$ is obtained from the solution of an integral equation containing matrix elements with the nonlocal complex potential. All details are given in Ref. [40]. The capture width $\left|V_{\mathbf{K}}^{(+)}\left(R_{v}\right)\right|^{2}$ can be calculated in the same way as the decay width, but without integration over $\hat{\mathbf{K}}$, therefore transformation (49) is unnecessary. For randomly oriented molecules we do the average over $\hat{\mathbf{R}}$.

The factor strongly influencing DEA cross sections is the Franck-Condon overlap between the initial vibrational state of the neutral molecule and the nuclear wave function for the negative-ion state. This overlap grows strongly when the negative-ion curve is shifted downward due to the polarization interaction between the negative ion and the medium. This effect was studied in our previous work $[8,22,23]$.

\section{B. Models for $\mathrm{CH}_{3} \mathrm{Cl}$ and $\mathrm{CF}_{3} \mathrm{Cl}$}

The theory developed is applied first to DEA to $\mathrm{CH}_{3} \mathrm{Cl}$ and $\mathrm{CF}_{3} \mathrm{Cl}$. These molecules exhibit very strong condensedmatter effects in the DEA process. According to previous theoretical results $[41,42]$, the gas-phase DEA cross section for $\mathrm{CH}_{3} \mathrm{Cl}$ at room temperature does not exceed 4 $\times 10^{-22} \mathrm{~cm}^{2}$, whereas corresponding values for molecules physisorbed on a $\mathrm{Kr}$ film and imbedded in a bulk $\mathrm{Kr}$ are $1.2 \times 10^{-17}$ and $1.0 \times 10^{-16} \mathrm{~cm}^{2}$, respectively, with the peak positions 0.5 and $0.2 \mathrm{eV}[8,22,23]$.

There are two gas-phase measurements of DEA to $\mathrm{CF}_{3} \mathrm{Cl}$ $[43,44]$, one using the swarm method and the other the beam method. Although there is some discrepancy between them, 
at room temperature both give the peak value about 2 $\times 10^{-18} \mathrm{~cm}^{2}$ with the position of the peak at about $1.7 \mathrm{eV}$. The latter is close to the value of the vertical attachment energy $E_{\mathrm{VAE}}=1.83 \mathrm{eV}$ obtained by the electron transmission spectroscopy method [45]. The DEA peak is not very strongly shifted relative to this value because the resonance is rather narrow. Measurements of the DEA to $\mathrm{CF}_{3} \mathrm{Cl}$ on the surface gives the peak value $0.4 \times 10^{-16} \mathrm{~cm}^{2}$ at $E=0.78 \mathrm{eV}$ $[9,23]$. When $\mathrm{CF}_{3} \mathrm{Cl}$ molecules are sandwiched between two $\mathrm{Kr}$ films, the corresponding peak value is $2.1 \times 10^{-16} \mathrm{~cm}^{2}$ at $E=0.60 \mathrm{eV}$ [23]. The enhancement is substantial, although not as significant as for $\mathrm{CH}_{3} \mathrm{Cl}$.

DEA to the $\mathrm{CH}_{3} \mathrm{Cl}$ and $\mathrm{CF}_{3} \mathrm{Cl}$ molecules is caused by the capture into the lowest unoccupied molecular orbital (LUMO) of the $a_{1}$ symmetry. Experimental data [46,47] on resonant vibrational excitation of these molecules indicate that the differential cross section at low energies is close to isotropic. Deviations from isotropy can be explained by the effects of the long-range dipolar interaction [41]. The $p$-wave contribution is suppressed in the low-energy capture amplitude because of the repulsive centrifugal barrier. In addition, at present we do not have information how these molecules are oriented on surfaces or in bulk in corresponding experiments. Therefore in our first application of the theory, as in our previous gas-phase calculations $[41,42,48]$, we will assume the $s$-wave dominance for gas-phase scattering. With this assumption the resonance width is independent of the molecular orientation, although comparison of Eq. (53) with Eq. (57) and Eq. (54) with Eq. (58) shows that averaging over orientations for the general case of an $l$-mixed resonance is not equivalent to the $l_{r}=0$ case.

For our model studies we use potential curves parametrized in the Morse form as described in our previous $R$-matrix calculations, Refs. $[41,42,48]$. However, the previously obtained curves should be modified since the resonance shift is different in $R$-matrix and nonlocal-complexpotential formalisms. The $R$-matrix shift is obtained from the logarithmic derivative of the electron wave function and the $R$-matrix surface amplitude [24,25]. The gas-phase calculations show that the $R$-matrix shift function is quite small, and does not exceed $0.3 \mathrm{eV}$ in absolute magnitude for $|E|$ $<2 \mathrm{eV}$, whereas the nonlocal shift function, Eq. (60), is much bigger reaching the value $-1.5 \mathrm{eV}$ at zero energy.

Since the diabatic state of the scattering theory and the resonance shift are not observable quantities, there is some arbitrariness in the choice of the diabatic state, although in an exact theory the final results for observable quantities, such as the resonance width and the scattering cross sections, should not depend on the choice of the diabatic state. In the present work we base our choice of the diabatic state on results of $a b$ initio calculations of adiabatic energies for $\mathrm{CH}_{3} \mathrm{Cl}[42]$ and $\mathrm{CF}_{3} \mathrm{Cl}[48]$ and experimental data [46,47] on the excitation of symmetric stretch vibrations $\nu_{3}$ in these molecules. The latter were also used to obtain the gas-phase resonance width in Refs. [42,48], which remains unchanged in the present work.

For surface and bulk calculations the negative-ion curve is shifted downwards by $0.72 \mathrm{eV}$ for the surface case and by $1.15 \mathrm{eV}$ for the bulk case in accordance with the results of the calculations of polarization energy for the $\mathrm{Kr}$ film [35].

\section{Image states, image-potential resonances, and threshold effects}

It is well known that insulators can support image states due to the image-potential interaction between the electron and the surface on the vacuum side [34]. The dielectric films are usually deposited on a metal substrate, and the latter increases the strength of the image potential (see, e.g., Refs. [49-52]). The coupling of the resonance anion states with the image states can strongly increase the efficiency of inelastic processes on the surface [16]. Nagesha and Sanche [10] observed tremendous increase in near-zero-energy electron trapping by adsorbed molecules at the surface of glassy $n$-hexane $(\mathrm{nHg})$. The conduction band edge for $n \mathrm{Hg}$ films lies $0.8 \mathrm{eV}$ above the vacuum level and this creates a favorable condition for formation of the image states. In contrast, in $\mathrm{Kr}$ films the bottom of the conduction band lies at $0.25 \mathrm{eV}$ below the vacuum level, and the enhancement is not observed. As we saw above, the image states are possible in $\mathrm{Kr}$ films too because of the different values of the normal energy components in vacuum and the film. However, they are very weakly bound, therefore their contribution to the decay width, Eq. (42), is expected to be small, as is confirmed by calculations. Moreover, their influence on the DEA process can be only adverse in the present formalism. Nagesha and Sanche [10] suggested that the mechanism for DEA enhancement involves initial electron capture into an image state due to electron-surface scattering with the following electron transfer to anionic state. This process is not incorporated into the present theory.

The image states have some similarities with Rydberg resonances important in dissociative recombination [53]. However, the image states are bound only along the $z$ axis and remain the plane waves in the direction parallel to the surface. Therefore they should be treated as a part of continuum as suggested by Eq. (42). Comparison of Eq. (52) with Eq. (50) indicates that the coupling of these states to the anion state increases substantially at low energies since the width $\Gamma^{(s)}$ does not contain the factor $K$ typical for scattering states. However, for a $\mathrm{Kr}$ surface the coupling remains weak even for low energies.

The presence of the image-charge potential leads also to another effect: oscillations of the reflection coefficient $R^{(-)}$ occur as a function of energy and, accordingly, oscillations in $\Gamma^{(-)}(E)$. Physically these features can be interpreted as image-charge-potential resonances for the perpendicular energy component below the vacuum level. This apparently corresponds to the total internal reflection for the minus solution. From condition (26) we obtain that the total internal reflection is possible if

$$
E<-\epsilon_{0}\left[\frac{m}{m^{*} \sin ^{2} \theta_{k}}-1\right]^{-1} .
$$

Therefore the maximum possible energy for which imagecharge resonances are observed is

$$
E_{\max }=-\frac{\epsilon_{0} m^{*}}{m-m^{*}} .
$$

For the $\mathrm{Kr}$ film this condition gives $E_{\max }=0.18 \mathrm{eV}$. Apparently, if $m^{*}<m$, the Coulomb oscillations in the width are 


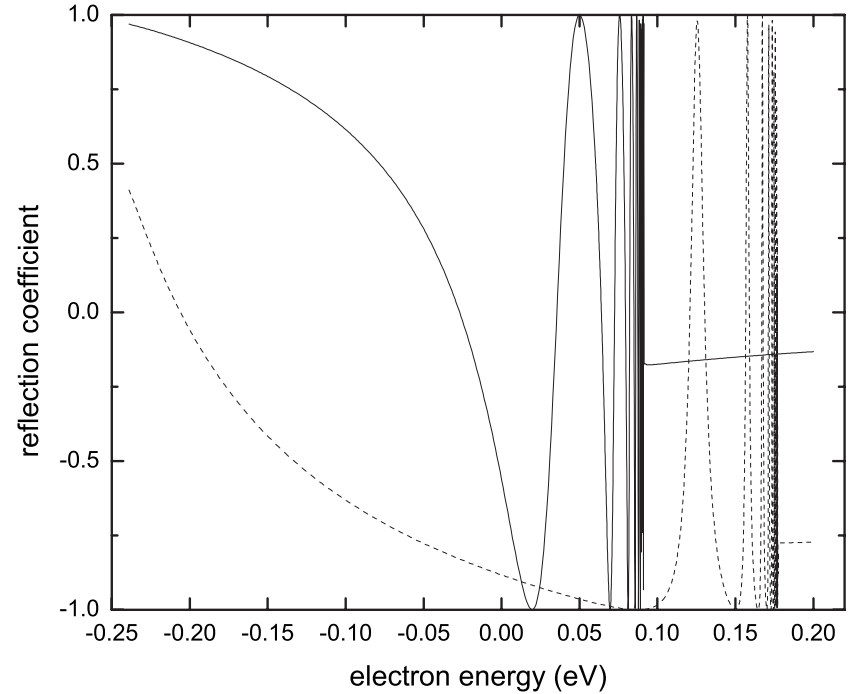

FIG. 2. Real part of the reflection coefficient $R^{(-)}$as a function of energy for $\cos \theta_{k}=0.6$ (solid line) and 0.1 (dashed line).

possible only if the bottom of the conduction band lies below the vacuum level. Note that these oscillations are not related to the image surface states since the corresponding wave function is not bounded from the medium side.

Figure 2 illustrates the behavior of the reflection coefficient $R^{(-)}$as a function of energy for two values of $\cos \theta_{k}, 0.6$ and 0.1 ( or $\theta_{k}=53^{\circ}$ and $84^{\circ}$ ). The energy given by the condition (62) is the accumulation point for the Coulomb resonances. This results in oscillatory behavior of the resonance width $\Gamma$ and shift $\Delta$ as functions of electron energy. In Fig. 3 we present the near-threshold surface width for $\mathrm{CH}_{3} \mathrm{Cl}$. Although the resonance width is nonzero even at negative energies (above $\epsilon_{0}$ ), the energy region where the width is mostly affected by the Coulomb resonances lies between 0 and $0.18 \mathrm{eV}$.

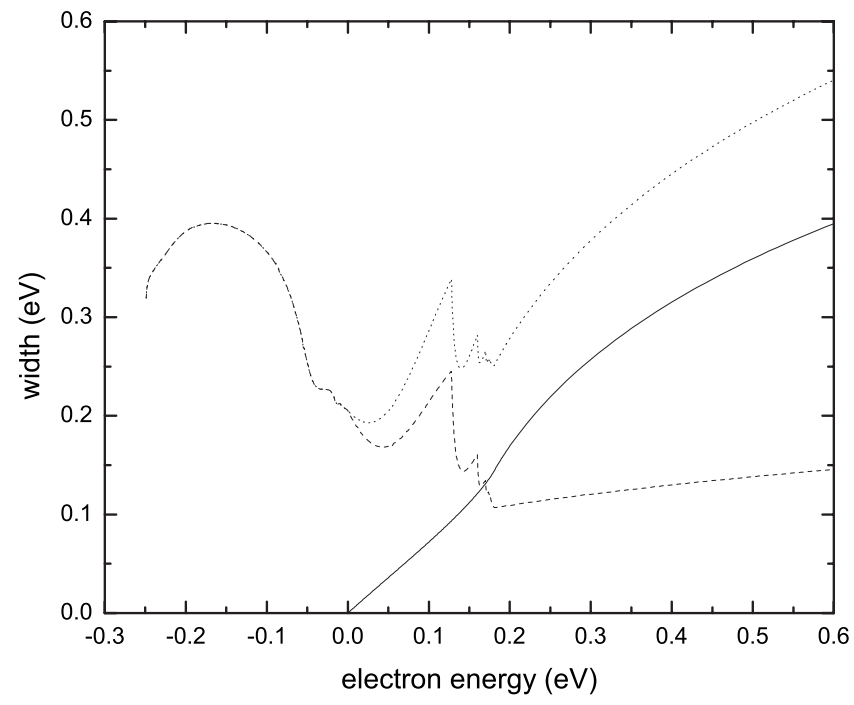

FIG. 3. Near-threshold behavior of the resonance width for the $\mathrm{CH}_{3} \mathrm{Cl}$ molecule on the $\mathrm{Kr}$-film surface at the equilibrium internuclear separation: $\Gamma^{(+)}$contribution (solid line), $\Gamma^{(-)}$contribution (dashed line), and the total width (dotted line).
Another interesting feature noticeable in Fig. 3 is the finite width at the threshold energy, $E=\epsilon_{0}$. At first glance this is not surprising since the gas-phase width is finite at the threshold for molecules with a supercritical permanent dipole moment [54]. However, inspection of Eq. (58) for $\Gamma^{(-)}$leads to a different conclusion. Equation (27) for the reflection coefficient indicates that $R^{(-)} \rightarrow-1+O(k)$ for $k \rightarrow 0$, as should be. Therefore the surface width should be suppressed by the factor $k^{2}$ as compared to the gas-phase width. However, calculations for $\mathrm{Kr}$ show that the derivative of the Coulomb function $\chi_{-K^{\perp}}^{\prime}$ accidentally passes through 0 very close to the bottom of the conduction band, therefore $R^{(-)}$is nonzero very close to the threshold. This phenomenon can be considered as a threshold image-potential resonance.

Let us look now at the threshold behavior of $\Gamma^{(+)}$. In this case the threshold corresponds to $E=0$. Equation (57) indicates that this behavior is determined by the threshold behavior of the transmission coefficient $T_{\mathbf{K}}^{(+)}$. According to Eq. (23), $T^{(+)} \propto K^{1 / 2}$ at $K \rightarrow 0$, where we have used the known expression for the Coulomb functions at $K \rightarrow 0$, [32]:

$$
F_{0}(r)=(\pi K r)^{1 / 2} J_{1}(\sqrt{2 C r}), \quad G_{0}(r)=-(\pi K r)^{1 / 2} Y_{1}(\sqrt{2 C r}),
$$

where $J_{1}$ and $Y_{1}$ are Bessel and Neumann functions. We conclude that $\Gamma^{(+)}$is proportional to $K^{2}$, or to the electron energy above the vacuum level. This behavior can also be observed in Fig. 3. Note that in the absence of the imagecharge-potential interaction $T^{(+)}$would be proportional to $K$ and $\Gamma^{(+)}$to $K^{3}$. This threshold behavior is essentially different from what is usually observed in standard theory of scattering by targets in the gas phase with the scattering wave description in spherical coordinates. Indeed, according to the Wigner threshold law [55] the inelastic scattering cross section, or the decay width, is finite at the threshold in the presence of the attractive Coulomb potential or dipolar potential with supercritical dipole moment [54], and is proportional to $K$ in the absence of the long-range interaction. The presence of the surface changes the threshold behavior. The threshold behavior of the inelastic cross section (or the decay width) is basically determined by two factors: the square of the wave function in the final state and the final density of states. For scattering by molecules in a vacuum the wave function is finite at the threshold in the short-range case and is proportional to $K^{-1 / 2}$ in the Coulomb case, whereas the density of final states is proportional to $K$. However, for scattering by targets on surfaces the wave function is proportional to $K$ in the short-range case and to $K^{1 / 2}$ in the Coulomb case, leading to the threshold behavior discussed above. Note that this qualitative change in the dependence of the wave function on $K$ is compatible with the correct threshold laws for scattering in one-dimensional systems [56] and scattering in the presence of an external magnetic field [57].

\section{Results for $\mathrm{CH}_{3} \mathrm{Cl}$ and $\mathrm{CF}_{3} \mathrm{Cl}$ and discussion}

In Fig. 4 we compare the gas-phase width with the width for a physisorbed molecule $\left(r_{s}=0\right)$ and for a molecule buried under the surface at a distance 50 a.u. in the (111) direction 


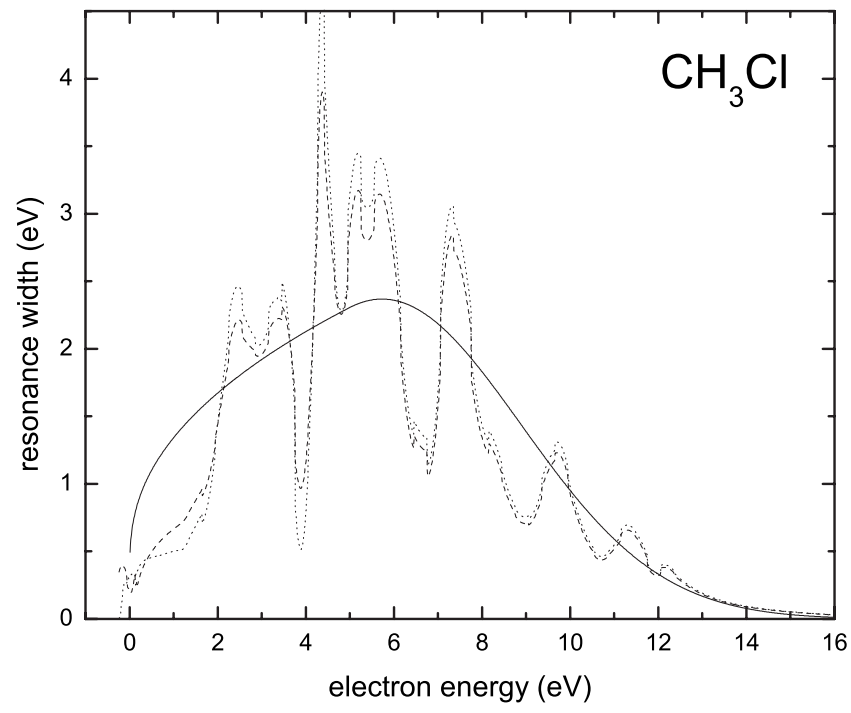

FIG. 4. Resonance width for the $\mathrm{CH}_{3} \mathrm{Cl}$ molecule at the equilibrium internuclear separation: solid line, gas-phase width; dashed line, molecules at the surface of the $\mathrm{Kr}$ film; dotted line, molecules buried under the surface at the distance 50 a.u.

of the lattice that corresponds to eight monolayers of $\mathrm{Kr}$ from the interface. We observe a substantial reduction of the width in the near-threshold region due to the medium effect. This is mainly due to the lower density of states in the medium, which is proportional to the effective mass $m^{*}$ in the near-threshold region.

Note that the width is nonzero below the vacuum level down to the bottom of the conduction band, since the electron can escape into the medium at $E>\epsilon_{0}$ even at negative energies. Accordingly, thresholds for vibrational excitation channels are redshifted by $\epsilon_{0}$. This leads to the increase in the negative-ion decay probability. However, this effect is small compared to those leading to the reduction of the decay width.

Another feature found in the calculations is the weak dependence of the width on $r_{s}$ for $r_{s}$ exceeding 20 a.u. In contrast, the experimental data on DEA to $\mathrm{CH}_{3} \mathrm{Cl}$ adsorbed on a $\mathrm{Kr}$ surface indicate a strong dependence of the DEA cross section on $r_{s}$ for film thicknesses less than 20 monolayers (125 a.u.) [8,23]. It is well known, however, that this dependence is due to the effect of the metal substrate [8]. Typically for a fixed film thickness, the greater is the distance $r_{s}$ of the molecule from the interface, the closer it is to the metal substrate. However, the metal effects are not included in the present theory.

In Fig. 5 we present the resonant shift calculations for the equilibrium internuclear separation. The relatively low value of the bulk width in the near-threshold region is reflected in the low values of the shift at low energies.

In Fig. 6 we present cross sections for DEA to the ground vibrational state of methyl chloride calculated with the use of the nonlocal complex potential theory incorporating the decay width obtained as described above. The gas-phase cross sections are very small and barely exceed $10^{-21} \mathrm{~cm}^{2}$. We should stress, however, that they are significantly higher than those obtained from the $R$-matrix calculation $[41,42]$ and are

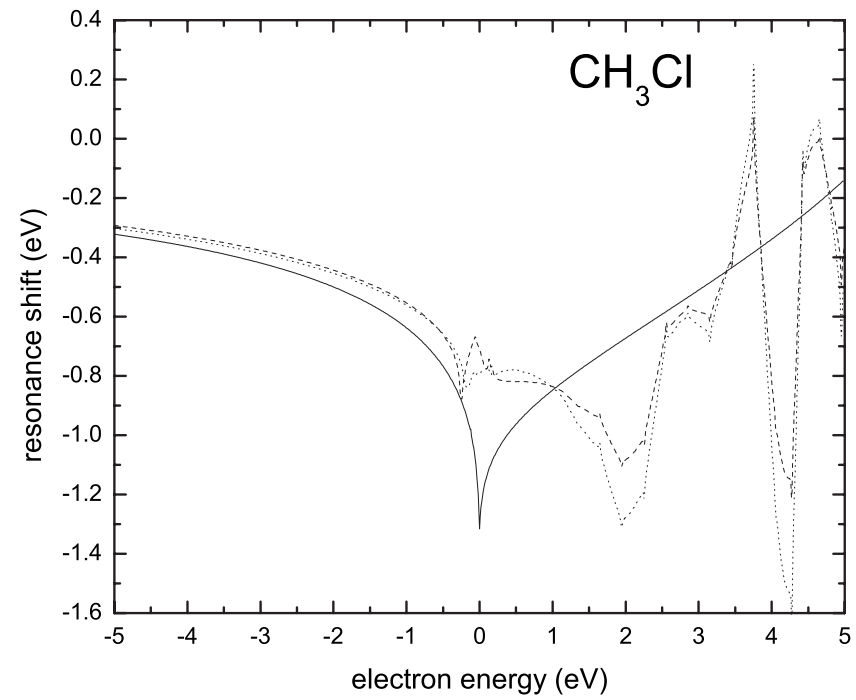

FIG. 5. Resonance shift for the $\mathrm{CH}_{3} \mathrm{Cl}$ molecule at the equilibrium internuclear separation. The curve notation is the same as in Fig. 4.

closer to the experimental upper bound at room temperature, $2 \times 10^{-21} \mathrm{~cm}^{2}[58]$, although both calculations are based on the same adiabatic potential energy curves and experimental information on vibrational excitation cross sections. This high sensitivity of the DEA cross sections to theoretical model is typical when the cross sections are very small. For higher temperatures, when the cross sections are significantly larger, both theoretical approaches become compatible and are in agreement with experimental data [42]. However, in the present work we are interested in cold molecules since in experiments they are deposited on $\mathrm{Kr}$ film at cryogenic temperatures.

The gas-phase cross sections exhibit peaks at thresholds for vibrational excitation of symmetric stretch $\mathrm{C}-\mathrm{Cl}$ vibrations. They are due to vibrational Feshbach resonances analyzed in detail in Refs. $[59,60]$. Some threshold structures are noticeable in the surface cross sections as well. However,

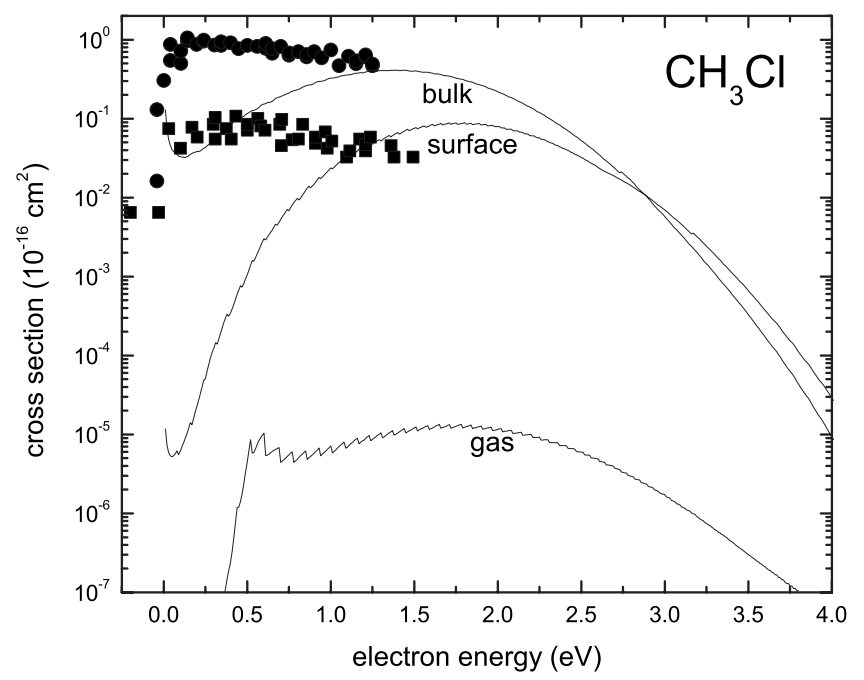

FIG. 6. DEA cross sections for the $\mathrm{CH}_{3} \mathrm{Cl}$ molecule. Experimental data: squares, surface; circles, bulk. 
their nature is different. Whereas in the gas phase the resonances appear due to the long-range (dipolar and polarization) interaction between the electron and the molecule, in the process at the surface the resonances are caused by the image-charge interaction as discussed in Sec. IV C.

The surface effects lead to a four orders of magnitude increase of cross sections as compared to the gas phase, and further one order of magnitude increase is observed for the bulk case. The obtained surface and bulk cross sections are bigger than those from our previous $R$-matrix calculations $[22,23]$ where the surface effects were accounted for mainly by the potential curve shifting and the incorporation of the density of states in the bulk. The inclusion of the correct geometry in the present calculations leads to further increase of the DEA cross sections. However, for the bulk case they still remain below the measured values by a factor of 2.5. A more striking disagreement with the experiment is observed in the peak position: the calculated curves peak at substantially higher energies than the experimental curves. It was argued in Ref. [23] that disagreement between theory and experiment for $\mathrm{CH}_{3} \mathrm{Cl}$ might be due to the nonuniform shift of the negative-ion curve. Due to modification of the interaction between the $\mathrm{Cl}^{-}$ion and the $\mathrm{CH}_{3}$ fragment by the medium, the anion curve is not simply uniformly shifted downwards, but becomes more repulsive. As was demonstrated in Ref. [23], this leads to a further increase of the DEA cross sections and shifting of the peak position to lower energies. Another drawback which might be even more important in the present studies is a possible inaccuracy in calculations of the resonance shift. Indeed, in the present nonlocal theory we need the width in the broad energy range in order to calculate the resonance shift. The approximation which was made in the width calculation for higher energies [effective mass approximation with the correction factor (47) for the density of states] might lead to an overestimate (underestimate in absolute magnitude) of the resonance shift. Indeed, from Fig. 5 we see that the surface and bulk shifts lie significantly higher than the gas-phase shift in the lowenergy region. As can be seen from Fig. 6, this leads to the fact, that the position of the peak in the DEA cross section virtually does not change when going from the gas phase to the surface (although it is shifted slightly towards lower energies for the bulk case). In contrast, the $R$-matrix calculations $[22,23]$ generate a significant shift in the peak position, mainly due to the polarization energy, since the resonance shift is small for both gas and surface cases. The sensitivity of the DEA cross section to the resonance shift is clearly seen in Fig. 6: the difference in the polarization shift in the surface and bulk calculations is only $0.43 \mathrm{eV}$, but this difference leads to the four orders difference in magnitude of the cross section in the low-energy region. This sensitivity suggests that the inaccuracy in the resonance shift calculation is the most likely reason for the wrong behavior of the calculated cross sections at $E<1 \mathrm{eV}$.

In Figs. 7-9 we present the resonance width, shift, and DEA cross sections for $\mathrm{CF}_{3} \mathrm{Cl}$. The results for the width and shift look qualitatively the same as those for $\mathrm{CH}_{3} \mathrm{Cl}$. However, in contrast to the $\mathrm{CH}_{3} \mathrm{Cl}$ case, the calculated cross sections are greater than measured, especially for molecules at the surface, where disagreement reaches a factor of 5. How-

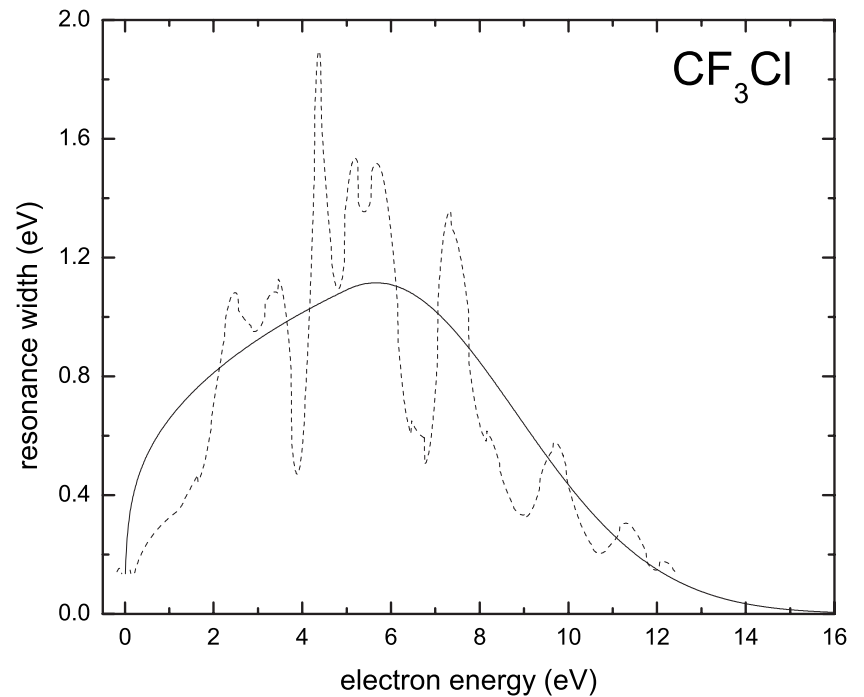

FIG. 7. Resonance width for the $\mathrm{CF}_{3} \mathrm{Cl}$ molecule at the equilibrium internuclear separation. Solid line, gas-phase width; dashed line, molecules at the surface of the $\mathrm{Kr}$ film.

ever, there is much better agreement in peak positions. Overall, the results show much better agreement with the experiment than previous $R$-matrix calculations [23]. However, qualitatively the situation is similar: for $\mathrm{CH}_{3} \mathrm{Cl}$ the theoretical results are too small and for $\mathrm{CF}_{3} \mathrm{Cl}$ they are too big. Since the velocity of $\mathrm{Cl}^{-}$is greater in the DEA process with $\mathrm{CF}_{3} \mathrm{Cl}$ than that with $\mathrm{CH}_{3} \mathrm{Cl}$, it was suggested in Ref. [23] that the cage effect [61] might play an important role in the former case. The cage effect leads to the return of the $\mathrm{Cl}^{-}$fragment into the region where the anion $\mathrm{CF}_{3} \mathrm{Cl}$ is unstable and decreases its survival probability. Another mass-dependent effect is the excitation of the phonon modes in the $\mathrm{Kr}$ film by the $\mathrm{Cl}^{-}$fragment. Interaction between the resonantly trapped electrons and acoustical phonons might be important in lowenergy inelastic scattering [62]. Again, in the $\mathrm{CF}_{3} \mathrm{Cl}$ case

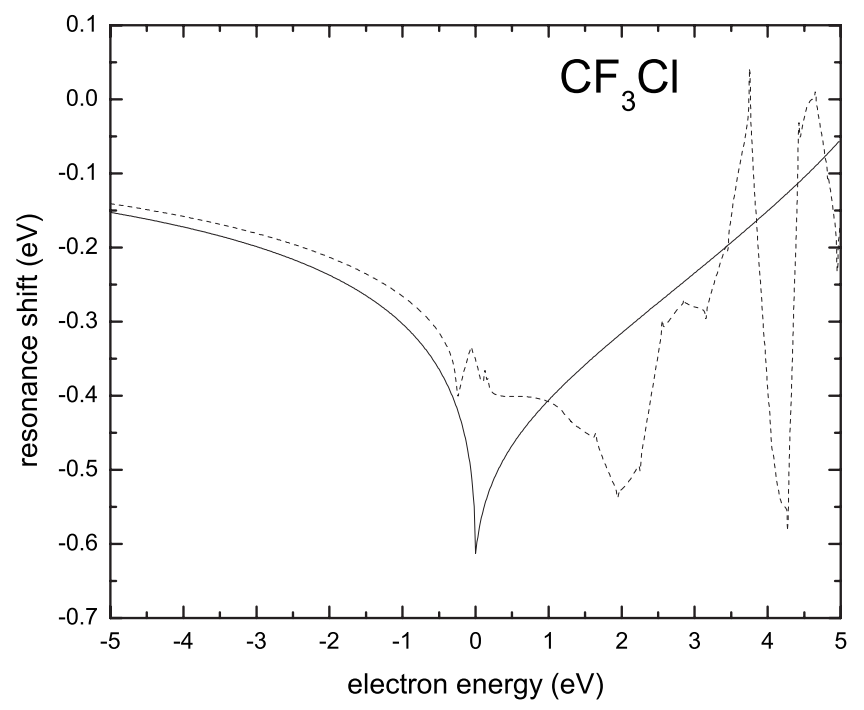

FIG. 8. Resonance shift for the $\mathrm{CF}_{3} \mathrm{Cl}$ molecule at the equilibrium internuclear separation. The curve notation is the same as in Fig. 7. 


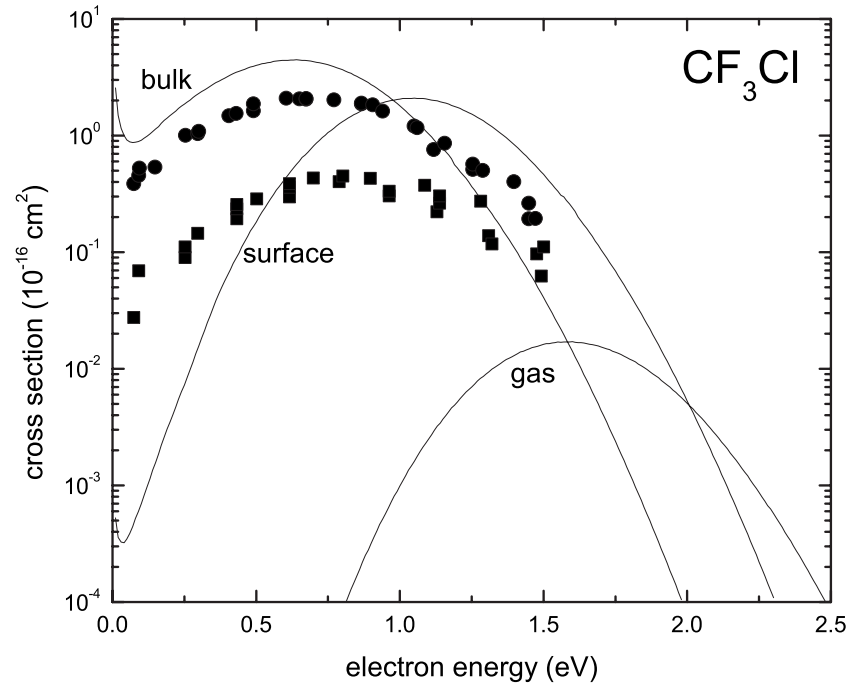

FIG. 9. DEA cross sections for the $\mathrm{CF}_{3} \mathrm{Cl}$ molecule. Experimental data: squares, surface; circles, bulk.

when the $\mathrm{Cl}^{-}$velocity is higher, this process is more efficient and can lead to lower DEA cross sections since inelastic anion-phonon interaction slows down the anion and decreases its survival probability. At the same time the probability of vibrationally inelastic process in electron-surface scattering is rather small, as has been established in electron energy-loss spectroscopy studies [63], therefore the electronphonon interaction in the initial state can be safely neglected in DEA calculations for physisorbed molecules. On the other hand, if the target molecule is buried deep in the medium, the electron energy loss before the attachment should be taken into consideration.

Note also that although the present calculations correspond to cryogenic temperatures relevant to experiments with rare-gas films, there are no major difficulties in extending them to a higher temperature region (provided that the crystal lattice still exists). The major effect here will be the increase of the population of vibrationally excited states of the target molecules, which in the case of $\mathrm{CH}_{3} \mathrm{Cl}$ and $\mathrm{CF}_{3} \mathrm{Cl}$ leads to a strong increase in DEA cross sections $[41,42,48]$. In contrast, the electronic structure parameters such as $\epsilon_{0}$ and $\kappa$ will not change significantly.

\section{CONCLUSION}

We have presented a theoretical description of surface and condensed matter effects in DEA based on nonlocal complex potential theory. The major step in the application of the theory is the conversion of the gas-phase resonance width into the condensed-matter width. In the first applications we have made several approximations in order to simplify this procedure. The most severe is the effective-mass approximation, which was corrected at higher electron energies in order to take into account the actual density of states in $\mathrm{Kr}$. Although we did some investigation of the role of the image states, it was shown that they play a very insignificant role in thick $\mathrm{Kr}$ films, and they were neglected in present calculations. In other dielectric media, however, particularly those exhibiting a surface barrier, these states might play a significant role $[10,16]$, and for this case the present theory should be developed further to take into account the image states as intermediaries in the anion formation.

The calculations of DEA to $\mathrm{CH}_{3} \mathrm{Cl}$ and $\mathrm{CF}_{3} \mathrm{Cl}$ molecules on surfaces and in the bulk of $\mathrm{Kr}$ films demonstrate that the present theory reproduces the major features observed in experiments: strong enhancement of DEA at the surface and in the medium as compared to the gas phase and the shifting of the peak position towards lower energies. However, quantitatively this shift is too small for $\mathrm{CH}_{3} \mathrm{Cl}$ as compared to the experimental data. This can be due to our underestimate (in absolute magnitude) of the resonance shift in the case of surface and medium. Detailed studies of the resonance width at higher energies beyond the effective mass approximation model are necessary to resolve this discrepancy. To explain too large values of DEA cross sections for $\mathrm{CF}_{3} \mathrm{Cl}$ at the $\mathrm{Kr}$ surface, the cage effect, and the anion-phonon interaction effects should be investigated.

\section{ACKNOWLEDGMENTS}

The author acknowledges numerous stimulating discussions with Laurent Caron, Andrey Kazansky, Wai-Ning Mei, and Leon Sanche. This work was supported by the National Science Foundation, Grant No. PHY-0354688.
[1] Linking the Gaseous and Condensed Phases of Matter, edited by L. G. Christophorou, E. Illenberger, and W. F. Schmidt, NATO ASI Series No. B326 (Plenum, New York, 1994).

[2] L. Sanche, Radiat. Phys. Chem. 32, 269 (1988).

[3] Q.-B. Lu and Th. E. Madey, Phys. Rev. Lett. 82, 4122 (1999).

[4] Q.-B. Lu and L. Sanche, Phys. Rev. Lett. 87, 078501 (2001).

[5] P. D. Burrow, K. Aflatooni, and G. A. Gallup, Environ. Sci. Technol. 34, 3368 (2000).

[6] R. E. Palmer and P. J. Rous, Rev. Mod. Phys. 64, 383 (1992).

[7] R. E. Palmer, Comments At. Mol. Phys. 30, 77 (1994).

[8] L. Sanche, A. D. Bass, P. Ayotte, and I. I. Fabrikant, Phys. Rev. Lett. 75, 3568 (1995).
[9] K. Nagesha and L. Sanche, Phys. Rev. Lett. 78, 4725 (1997).

[10] K. Nagesha and L. Sanche, Phys. Rev. Lett. 81, 5892 (1998).

[11] F. Weik, E. Illenberger, K. Nagesha, and L. Sanche, J. Phys. Chem. B 102, 824 (1998).

[12] P. D. Burrow and K. Aflatooni, J. Chem. Phys. 116, 5471 (2002).

[13] Q.-B. Lu, A. D. Bass, and L. Sanche, Phys. Rev. Lett. 88, 147601 (2002).

[14] A. Srivastava and R. M. Osgood, Jr., J. Chem. Phys. 119, 10298 (2003).

[15] P. Tegeder, R. Balog, N. J. Mason, and E. Illenberger, Phys. Chem. Chem. Phys. 7, 685 (2005). 
[16] P. J. Rous, Phys. Rev. Lett. 74, 1835 (1995).

[17] P. J. Rous, Phys. Rev. Lett. 83, 5086 (1999).

[18] D. C. Marinica, D. Teillet-Billy, J.-P. Gauyacq, M. Michaud, and L. Sanche, Phys. Rev. B 64, 085408 (2001).

[19] D. C. Marinica, D. Teillet-Billy, and J.-P. Gauyacq, Phys. Rev. B 71, 115438 (2005).

[20] D. C. Marinica, C. Ramseyer, D. Teillet-Billy, and J. P. Gauyacq, Surf. Sci. 600, 803 (2006).

[21] P. Ayotte, J. Gamache, A. D. Bass, L. Sanche, and I. I. Fabrikant, J. Chem. Phys. 106, 749 (1997).

[22] I. I. Fabrikant, K. Nagesha, R. Wilde, and L. Sanche, Phys. Rev. B 56, R5725 (1997).

[23] K. Nagesha, I. I. Fabrikant, and L. Sanche, J. Chem. Phys. 114, 4934 (2001).

[24] I. I. Fabrikant, Comments At. Mol. Phys. 24, 37 (1990).

[25] I. I. Fabrikant, Phys. Rev. A 43, 3478 (1991).

[26] S. Y. Tong, in Progress in Surface Science, edited by S. G. Davisson (Pergamon, London, 1975), Vol. 7, p. 1; J. B. Pendry, Low-Energy Electron Diffraction (Academic Press, New York, 1971).

[27] N. F. Lane, Rev. Mod. Phys. 52, 29 (1980).

[28] T. F. O’Malley and H. S. Taylor, Phys. Rev. 176, 207 (1968).

[29] J. B. Pendry, in Structure of Surfaces, edited by M. A. Van Hove and S. Y. Tong, Springer Series in Surface Sciences (Springer-Verlag, Berlin, 1984), p. 124.

[30] J. A. Strozier, Jr. and R. O. Jones, Phys. Rev. B 3, 3228 (1971).

[31] Rare Gas Solids, edited by M. L. Klein and J. A. Venables (Academic Press, London, 1976), Vols. 1 and 2, p. 522.

[32] M. Abramowitz and I. S. Stegun, Handbook of Mathematical Functions (Dover, New York, 1972).

[33] D. J. BenDaniel and C. B. Duke, Phys. Rev. 152, 683 (1966).

[34] M. W. Cole, Phys. Rev. B 2, 4239 (1970); 3, 4418 (1971).

[35] M. Michaud and L. Sanche, J. Electron Spectrosc. Relat. Phenom. 51, 237 (1990).

[36] M. Michaud, P. Cloutier, and L. Sanche, Phys. Rev. B 44, 10485 (1991).

[37] M. Michaud, L. Sanche, T. Goulet, and J.-P. Jay-Gerin, Phys. Rev. Lett. 66, 1930 (1991).

[38] N. C. Bacalis, D. A. Papaconstantopoulos, and W. E. Pickett, Phys. Rev. B 38, 6218 (1988).

[39] W. Domcke, Phys. Rep. 208, 97 (1991).

[40] S. A. Kalin and A. K. Kazansky, J. Phys. B 23, 4377 (1990).
[41] I. I. Fabrikant, J. Phys. B 27, 4325 (1994).

[42] D. M. Pearl, P. D. Burrow, I. I. Fabrikant, and G. A. Gallup, J. Chem. Phys. 102, 2737 (1995).

[43] S. M. Spyrou and L. G. Christophorou, J. Chem. Phys. 82, 2620 (1982).

[44] T. Underwood-Lemons, T. J. Gergel, and J. H. Moore, J. Chem. Phys. 102, 119 (1995).

[45] K. Aflatooni and P. D. Burrow, Int. J. Mass. Spectrom. 205, 149 (2001).

[46] A. Mann and F. Linder, J. Phys. B 25, 1621 (1992).

[47] X. Shi, V. K. Chan, G. A. Gallup, and P. D. Burrow, J. Chem. Phys. 104, 1855 (1996).

[48] R. S. Wilde, G. A. Gallup, and I. I. Fabrikant, J. Phys. B 32, 663 (1999).

[49] B. Trninić-Radja, M. Šunjić, and Z. Lenac, Phys. Rev. B 40, 9600 (1989).

[50] M. Wolf, E. Knoesel, and T. Hertel, Phys. Rev. B 54, R5295 (1996).

[51] J. D. McNeill, R. L. Lingle, Jr., R. E. Jordan, D. F. Padowitz, and C. B. Harris, J. Chem. Phys. 105, 3883 (1996).

[52] D. C. Marinica, C. Ramseyer, A. G. Borisov, D. Teillet-Billy, J. P. Gauyacq, W. Berthold, P. Feulner, and U. Höfer, Phys. Rev. Lett. 89, 046802 (2002).

[53] W. Domcke, J. Phys. B 16, 359 (1983).

[54] I. I. Fabrikant, Sov. Phys. JETP 46, 693 (1977).

[55] E. P. Wigner, Phys. Rev. 73, 1002 (1948).

[56] H. R. Sadeghpour, J. L. Bohn, M. J. Cavagnero, B. D. Esry, I. I. Fabrikant, J. H. Macek, and A. R. P. Rau, J. Phys. B 33, R93 (2000).

[57] C. W. Clark, Phys. Rev. A 28, 83 (1983).

[58] S. C. Chu and P. D. Burrow, Chem. Phys. Lett. 172, 17 (1990).

[59] A. Schramm, I. I. Fabrikant, J. M. Weber, E. Leber, M.-W. Ruf and H. Hotop, J. Phys. B 32, 2153 (1999).

[60] R. S. Wilde, G. A. Gallup and I. I. Fabrikant, J. Phys. B 33, 5479 (2000).

[61] R. Schriever, M. Chergui, Ö. Ünal, N. Schwentner, and V. Stepanenko, J. Chem. Phys. 93, 3245 (1990).

[62] D. L. Mills, Phys. Rev. B 45, 36 (1992).

[63] S. Y. Tong, C. H. Li, and D. L. Mills, Phys. Rev. Lett. 44, 407 (1980); M.-L. Xu, B. M. Hall, S. Y. Tong, M. Rocca, H. Ibach, S. Lehwald, and J. E. Black, ibid. 54, 1171 (1985). 